\title{
Preface: Information and Diffusion
}

Volume 3 of the book focuses on the third core factor, namely, "information and diffusion," which consists of four chapters. Chapter 1 is about the information retrieval for social networks, Chapter 2 is about the rules of information diffusion in social networks, Chapter 3 is the topic discovery and evolution, and Chapter 4 is about the influence maximization algorithms.

The following experts and scholars who participated in the data collection, content arrangement, and achievement contribution of this volume are sincerely appreciated: Zhaoyun Ding, Xiaomeng Wang, Bin Wang, Yezheng Liu, Xiaodong Liu, Shenghong Li, Aiping Li, Lei Li, Shiyu Du, Peng Wu, Xiuzhen Chen, Wei Chen, Yang Yang, Lumin Zhang, Peng Shi, and Yuanchun Jiang.

Thanks to Associate Professor Shudong Li for the careful coordination and arrangement for writing this volume, and also to Weihong Han and Shuqiang Yang for reviewing and proofreading. 
